\title{
Inactivation of Vibrio parahaemolyticus and Vibrio vulnificus in Phosphate-Buffered Saline and in Inoculated Whole Oysters by High-Pressure Processing
}

\author{
JAHEON KOO,${ }^{1} \dagger$ MICHAEL L. JAHNCKE, ${ }^{1 *}$ PAUL W. RENO,${ }^{2}$ XIAOPEI HU, ${ }^{3}$ \\ AND PARAMESWARAKUMAR MALLIKARJUNAN ${ }^{3}$
}

\begin{abstract}
${ }^{1}$ Virginia Seafood Agricultural Research and Extension Center, Virginia Polytechnic Institute and State University, Virginia Sea Grant Program, 102 South King Street, Hampton, Virginia 23693; ${ }^{2}$ Department of Microbiology, Oregon State University, Hatfield Marine Science Center, 2030 S.E. Marine Science Drive, Newport, Oregon 97365; and ${ }^{3}$ Department of Biological Systems Engineering, Virginia Polytechnic Institute and State University, 200 Seitz Hall, Blacksburg, Virginia 24060, USA
\end{abstract}

MS 04-488: Received 29 October 2004/Accepted 5 July 2005

\begin{abstract}
Inactivation studies for Vibrio parahaemolyticus TX-2103 (serotype O3:K6) and Vibrio vulnificus MO-624 (clinical isolate) were conducted in phosphate-buffered saline (PBS) and in inoculated oysters under high-pressure processing conditions. $V$. parahaemolyticus was more resistant than $V$. vulnificus in PBS at all pressures and times. A 6-log reduction of $V$. parahaemolyticus and $V$. vulnificus in PBS at $241 \mathrm{MPa}$ required 11 and $5 \mathrm{~min}$, respectively, which included a 3-min pressure come-up time. A 4.5-log reduction of $V$. parahaemolyticus in oysters at $345 \mathrm{MPa}$ required $7.7 \mathrm{~min}$, which included a 6.7-min pressure come-up time. More than a 5.4-log reduction of $V$. vulnificus in oysters at $345 \mathrm{MPa}$ occurred during the 6-min pressure come-up time. Both V. parahaemolyticus and V. vulnificus in PBS and in oysters were reduced to nondetectable numbers at $586 \mathrm{MPa}$ during the 8- and 7-min pressure come-up times, respectively.
\end{abstract}

High-pressure processing (HPP) is a nonthermal technology that may cause only minor deleterious changes to sensory and nutritional characteristics of foods, but it can effectively eliminate or substantially reduce spoilage microorganism and can inactivate enzymes (13). HPP has been used to destroy yeast, molds, bacterial spores, and vegetative bacteria, including most infectious foodborne pathogens, in foods $(10,13)$. It has been effective in oysters for reducing total bacteria, including Vibrio spp. (1-3, 9, 11, 15). The use of HPP also reduces labor requirements in the oyster industry because it is an effective method for shucking (9).

Vibrio parahaemolyticus and Vibrio vulnificus are naturally present in waters where oysters are grown and harvested (4). Foodborne illnesses caused by V. parahaemolyticus and $V$. vulnificus are usually associated with consumption of raw or undercooked molluscan shellfish (5, 14). From 1997 through 1998, outbreaks from V. parahaemolyticus infection occurred in Washington, Texas, and New York (7). The V. parahaemolyticus infection outbreak in Texas was the first reported to be associated with the O3: K6 serotype in the United States (5, 7). Most reported cases of $V$. vulnificus infections are caused by consumption of molluscan shellfish harvested from coastal states from the Gulf of Mexico region (14). Approximately $40 \%$ of reported V. vulnificus illnesses are fatal (12).

\footnotetext{
* Author for correspondence. Tel: 757-727-4861; Fax: 757-727-4871; E-mail: mjahncke@vt.edu.

$\dagger$ Present address: Department of Agriculture, University of Arkansas at Pine Bluff, Mail Slot 4913, Pine Bluff, AR 71601, USA.
}

Vibrio cholerae $\mathrm{O} 1$ and non-O1, V. parahaemolyticus, $V$. vulnificus, $V$. hollisae, and $V$. mimicus in artificial seawater, phosphate-buffered saline (PBS), oyster homogenates, and whole oysters (Crassostrea virginica and Crassostrea gigas) were destroyed by HPP at pressure levels ranging from 200 to $345 \mathrm{MPa}(1-3)$. Calik et al. (2) reported slight differences in pressure resistance between environmental and clinical strains of $V$. parahaemolyticus in PBS and in oysters. Cook (3) also reported that clinical, environmental, and various food isolates of $V$. cholerae, $V$. parahaemolyticus, and $V$. vulnificus had different pressure resistances in PBS at 200 and $250 \mathrm{MPa}$. $D$-values of $V$. parahaemolyticus $\mathrm{O} 3: \mathrm{K} 6$ in $\mathrm{PBS}$ were more than twice as high as those for non-O3:K6 serotypes at $250 \mathrm{MPa}$ (3). Cook (3) reported that $225 \mathrm{MPa}$ for $3 \mathrm{~min}$ or $250 \mathrm{MPa}$ for 2 min was required for a 5-log reduction of $V$. vulnificus and $300 \mathrm{MPa}$ for $3 \mathrm{~min}$ was required for the same reduction in V. parahaemolyticus. Cook (3) also evaluated differences in inactivation of $V$. vulnificus in whole oysters versus oyster homogenates. No difference was found, and at $241 \mathrm{MPa}$ for 2 min there was more than a 4.8-log reduction of V. vulnificus in both whole oysters and in oyster homogenates (3).

In addition to destruction of pathogens, HPP is also an effective oyster shucking procedure. Optimum shucking pressures for Pacific oysters with minimal affect on outward appearance ranged from 240 to $275 \mathrm{MPa}$, with $100 \%$ shucking efficiency at $310 \mathrm{MPa}$ for $0 \mathrm{~min}$ (i.e., as soon as the pressure unit reaches the target pressure it is immediately depressurized) (9). López-Caballero et al. (11) reported that processing at $400 \mathrm{MPa}$ for $10 \mathrm{~min}$ at $7^{\circ} \mathrm{C}$ did 
not affect the appearance of the oyster meat. However, $\mathrm{He}$ et al. (9) reported that oysters processed at lower pressures maintained higher sensory quality during shelf life, but changes in color and other sensory characteristics occurred at higher pressures.

The present study was focused on survival profiles of $V$. parahaemolyticus and $V$. vulnificus in PBS and in whole Eastern oysters $(C$. virginica) at broader ranges of processing times and pressures than have been evaluated in previous studies $(2,3)$.

\section{MATERIALS AND METHODS}

Bacterial strains. Clinical isolates of $V$. parahaemolyticus TX-2103 (serotype O3:K6) and V. vulnificus MO-624 were obtained from the U.S. Food and Drug Administration (Dauphin Island, Ala.). Both strains were maintained at room temperature on $\mathrm{T}_{1} \mathrm{~N}_{1}$ agar slants ( $10 \mathrm{~g}$ of tryptone, $10 \mathrm{~g}$ of $\mathrm{NaCl}, 20 \mathrm{~g}$ of agar, and 1.0 liter of distilled water) under sterile mineral oil. Strains were streaked on tryptic soy agar plates containing $1 \% \mathrm{NaCl}$ (TSAS) and incubated overnight at $35^{\circ} \mathrm{C}$. An isolated colony of each strain was picked, inoculated into $\mathrm{T}_{1} \mathrm{~N}_{1}$ broth (10 g of tryptone, $10 \mathrm{~g}$ of $\mathrm{NaCl}$, and 1.0 liter of distilled water), and incubated overnight at $35^{\circ} \mathrm{C}$. A loop of culture was transferred to another fresh $\mathrm{T}_{1} \mathrm{~N}_{1}$ broth tube and incubated for $18 \mathrm{~h}$ at $35^{\circ} \mathrm{C}$ before use.

Preparation of pure culture sample in PBS. From each $V$. parahaemolyticus and $V$. vulnificus culture, $2 \mathrm{ml}$ was transferred separately into a regular polyester pouch (4 by 6 in. [10.2 by 15.2 cm], 2.5 mil thickness; Kapak Corporation, Minneapolis, Minn.) containing $18 \mathrm{ml}$ of PBS to obtain approximately $10^{7} \mathrm{CFU} / \mathrm{ml}$. The pouch was heat sealed with as few air bubbles as possible. Duplicate pouches were placed in other pouches $(6.5$ by 8 in. [16.5 by $20.3 \mathrm{~cm}]$ ) and heat sealed with $10 \mathrm{ml}$ of disinfectant (Lysol, Reckitt Benckiser, Slough, Berkshire, UK). The pouches were then placed in an insulated ice chest containing gel packs and shipped by overnight carrier to the Oregon State University (OSU) Department of Food Science and Technology Pilot Plant (Corvallis, Oreg.) for HPP treatment. Samples were pressure treated at the OSU Pilot Plant within $24 \mathrm{~h}$ after samples were packed and shipped out. After HPP treatment, the pouches were repacked in the same insulated ice chest containing gel packs and shipped by overnight carrier back to the Virginia Seafood Agricultural Research and Extension Center (VSAREC) where microbiological analyses were performed within $24 \mathrm{~h}$ after HPP treatment. Shipment temperatures were maintained at 5 to $10^{\circ} \mathrm{C}$.

Preparation and inoculation of Eastern oysters with $V$. parahaemolyticus and V. vulnificus. Fresh Eastern oysters were obtained from a local oyster supplier in Virginia. For the V. parahaemolyticus study, fresh oysters were shipped to the OSU Hatfield Marine Science Center (HMSC) for inoculation and microbiological analyses. Oysters were inoculated at the HMSC and then pressure treated at the OSU Pilot Plant. Pressure-treated oysters were analyzed at the HMSC. For the $V$. vulnificus study, oysters were inoculated at the VSAREC and shipped to the OSU Pilot Plant. After HHP processing, samples were shipped back to the VSAREC by overnight carrier for analysis.

Oysters were brushed and cleaned under running tap water. Thirty to 40 oysters were placed in an aquarium filled with 40 liters of seawater ( $25 \mathrm{ppt}$ salinity). On the first day, the oysters were fed with algae, and on the second day half of the seawater was replaced with fresh seawater. On the third day, the aquaria were placed in a class II biosafety cabinet (Labconco Corporation, Kansas City, Mo.), and $100 \mathrm{ml}$ of overnight cultures of V. para-
TABLE 1. Pressures and processing times used to treat Vibrio parahaemolyticus $T X-2103$ (serotype O3:K6) and V. vulnificus MO-624 (clinical isolate) in pure cultures in PBS and in whole inoculated oysters ${ }^{a}$

\begin{tabular}{ccc}
\hline & \multicolumn{2}{c}{ Processing time $(\min )^{b}$} \\
\cline { 2 - 2 } $\begin{array}{c}\text { Pressure } \\
(\mathrm{MPa})\end{array}$ & V. parahaemolyticus & V. vulnificus \\
\hline
\end{tabular}

207

$\begin{array}{lll}\text { PBS } & 0,4,8,12,16,20, \text { and } 22 & 0,2,3,4,6, \text { and } 9 \\ \text { Oyster } & \text { ND }\end{array}$

Oyster ND ND

241
PBS
$0,2,4,6,8$, and 10
$0,1,2$, and 3
Oyster ND
0,1 , and 2

276

$\begin{array}{lll}\text { PBS } & 0,1,2,3,4, \text { and } 5 & 0,1, \text { and } 2 \\ \text { Oyster } & 0,1,2,3,4,5 \text {, and } 6 & 0,1, \text { and } 2\end{array}$

Oyster $0,1,2,3,4,5$, and $6 \quad 0,1$, and 2

310

PBS $0,1,2$, and $3 \quad 0$ and 1

Oyster $\quad 0,1,2,3,4$, and $5 \quad 0,1$, and 2

345

PBS 0 and $1 \quad 0$

Oyster $0,1,2$, and $3 \quad 0$ and 1

379

PBS 0 and 1

Oyster 0 and $1 \quad 0$ and 1

586

PBS $0 \quad 0$

Oyster $0 \quad 0$

${ }^{a}$ Three to six replications of each condition were conducted in duplicate.

${ }^{b}$ Processing times do not include pressure come-up times, which ranged from 3 to $4 \mathrm{~min}$ for PBS and from 4 to $8 \mathrm{~min}$ for oysters. 0 min: when the pressure unit reaches the target pressure, the unit is immediately depressurized. ND, not determined.

haemolyticus or $V$. vulnificus was inoculated into each aquarium. The concentration of $V$. parahaemolyticus or $V$. vulnificus in the aquarium seawater was approximately $10^{6}$ to $10^{7} \mathrm{CFU} / \mathrm{ml}$. The next day, three or four inoculated oysters were placed into heavyduty pouches ( 8 by 12 in. [ 20.3 by $30.5 \mathrm{~cm}$ ], 4.5 mil thickness; Kapak) in duplicate. Duplicate pouches were then placed in another pouch containing $10 \mathrm{ml}$ of disinfectant and heat sealed. The same procedures used for shipping the pure culture samples were followed for the oyster samples. Inoculation aquaria were disinfected with 5,000 ppm chlorine for a minimum of $30 \mathrm{~min}$ before the seawater was discarded. All reusable laboratory utensils and disposable items were autoclaved after use.

Control procedures. A centrifuge tube filled with water was used as a temperature monitor during shipment. At receipt both in Oregon and Virginia, the temperature of the water in the centrifuge tube was checked to ensure that temperatures were maintained between 5 and $10^{\circ} \mathrm{C}$. Duplicate pouches containing cell suspensions of $V$. parahaemolyticus or $V$. vulnificus in PBS were used as controls. The control samples were not pressure treated. Control pouches were placed in the shipping container along with the test samples to determine whether shipping conditions were detrimental to survival of $V$. parahaemolyticus and $V$. vulnificus. 


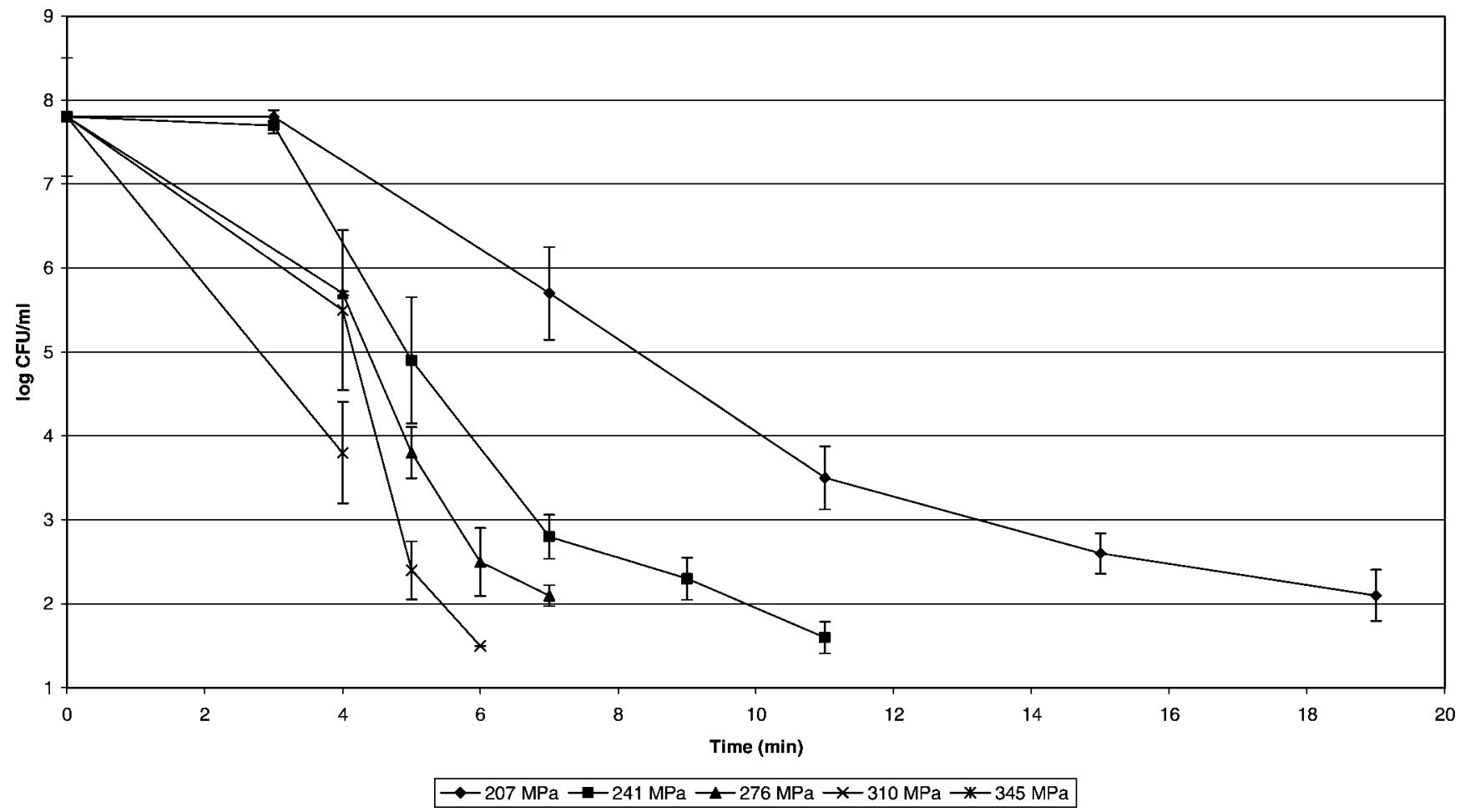

FIGURE 1. HPP inactivation of V. parahaemolyticus TX-2103 (serotype O3:K6) in PBS. Pressure come-up times are included in processing times.

High-hydrostatic-pressure processing. Pure cultures and inoculated oysters were treated with HPP at various pressures and times (Table 1) in a high-pressure machine (Engineered Pressure System, National Forge Company, Andover, Mass.) with a 22liter vessel. The samples were submerged in 50:50 mixtures of water and Houghto-safe 620 (glycol). A processing time of 0 min indicates that as soon as the pressure unit reached the target pressure it was immediately depressurized. Depressurization times were less than $2 \mathrm{~s}$ at all pressures. All samples were treated at $21^{\circ} \mathrm{C}$.

Microbiological analyses. For pure cultures in PBS, pouches were aseptically opened and aliquots were withdrawn and serially diluted. Approximately $5 \mathrm{ml}$ of sample was transferred into a sample cup and plated onto TSAS with a spiral plater (Microbiology International, Frederick, Md.). Plates were incubated at $35^{\circ} \mathrm{C}$ for 18 to $20 \mathrm{~h}$. For inoculated oysters, pouches were also aseptically opened, and the meat and liquid were placed into a blender jar (Waring, Torrington, Conn.). An equal volume of PBS was added, the oyster mixture was blended for $2 \mathrm{~min}$, and the homogenate was serially diluted. The hydrophobic grid membrane filter (HGMF) method was used to enumerate $V$. parahaemolyticus (2). The HGMF method is more specific for $V$. parahaemolyticus detection and enumeration than is the most-probable-number method (6). Sterile Millipore membrane filters (no. HAEG047AW, Fisher Scientific, Pittsburgh, Pa.) were placed on the surface of the filtration base, and the highest dilution was poured into the glass funnel and filtered with a vacuum pump. The membrane filter was then placed on a surface of plates (60-mm petri dish) with marine tryptic soy agar (MTSA; tryptic soy agar prepared in seawater with 25 ppt salinity). After enrichment for 4 to $6 \mathrm{~h}$ at $35^{\circ} \mathrm{C}$ on MTSA, the filter was transferred to either modified cellobiose polymyxin colistin (mCPC) agar plate or thiosulfate citrate bile salts sucrose (TCBS) agar plate for $V$. vulnificus or $V$. parahaemolyticus, respectively. The $\mathrm{mCPC}$ and TCBS plates were then incubated at $41^{\circ} \mathrm{C}$ for 18 to $24 \mathrm{~h}$. Aerobic plate counts (APCs) were determined on TSAS plates using the HGMF method after incubation at $35^{\circ} \mathrm{C}$ for $48 \mathrm{~h}$. Detection limits for the enumeration methods in pure cultures and in oysters were less than $20 \mathrm{CFU} /$ $\mathrm{ml}$ and $10 \mathrm{CFU} / \mathrm{g}$, respectively.

Decimal reduction times. For each pressure tested, a minimum of three to six replications were conducted. Each replication was conducted on separate days, and each replication was conducted in duplicate. Bacterial cultures were freshly prepared from the same slant for each HPP test. Pressure come-up times, which ranged from 3 to $4 \mathrm{~min}$ for PBS and 4 to $8 \mathrm{~min}$ for oysters, were added to processing times in the linear regression lines. The mean $D$-values for HPP treatments were calculated from the negative reciprocals of the slopes of the linear regression lines from the straight portions of the survival curves.

\section{RESULTS AND DISCUSSION}

Pure cultures. Evaluation of the control samples revealed no significant effect on bacterial counts before and after shipping and handling. In PBS, V. vulnificus MO-624 (clinical isolate) was more sensitive than $V$. parahaemolyticus TX-2103 (serotype 03:K6) at all pressures and times (Figs. 1 and 2). V. parahaemolyticus and V. vulnificus were proportionately more sensitive as the pressure increased. Average initial $V$. parahaemolyticus and $V$. vulnificus concentrations (up to six replications) was $7.8 \mathrm{log} \mathrm{CFU} / \mathrm{ml}$. The HPP times for $V$. parahaemolyticus were approximately two times longer than the times needed for $V$. vulnificus to achieve a 6-log reduction at 207 and $241 \mathrm{MPa}$ (Figs. 1 and 2). At $310 \mathrm{MPa}$ for $6 \mathrm{~min}$, including a 2-min pressure come-up time, $V$. parahaemolyticus was reduced by $6.3 \mathrm{log}$ units (Fig. 1). At pressures of $310 \mathrm{MPa}$ and higher for 5 


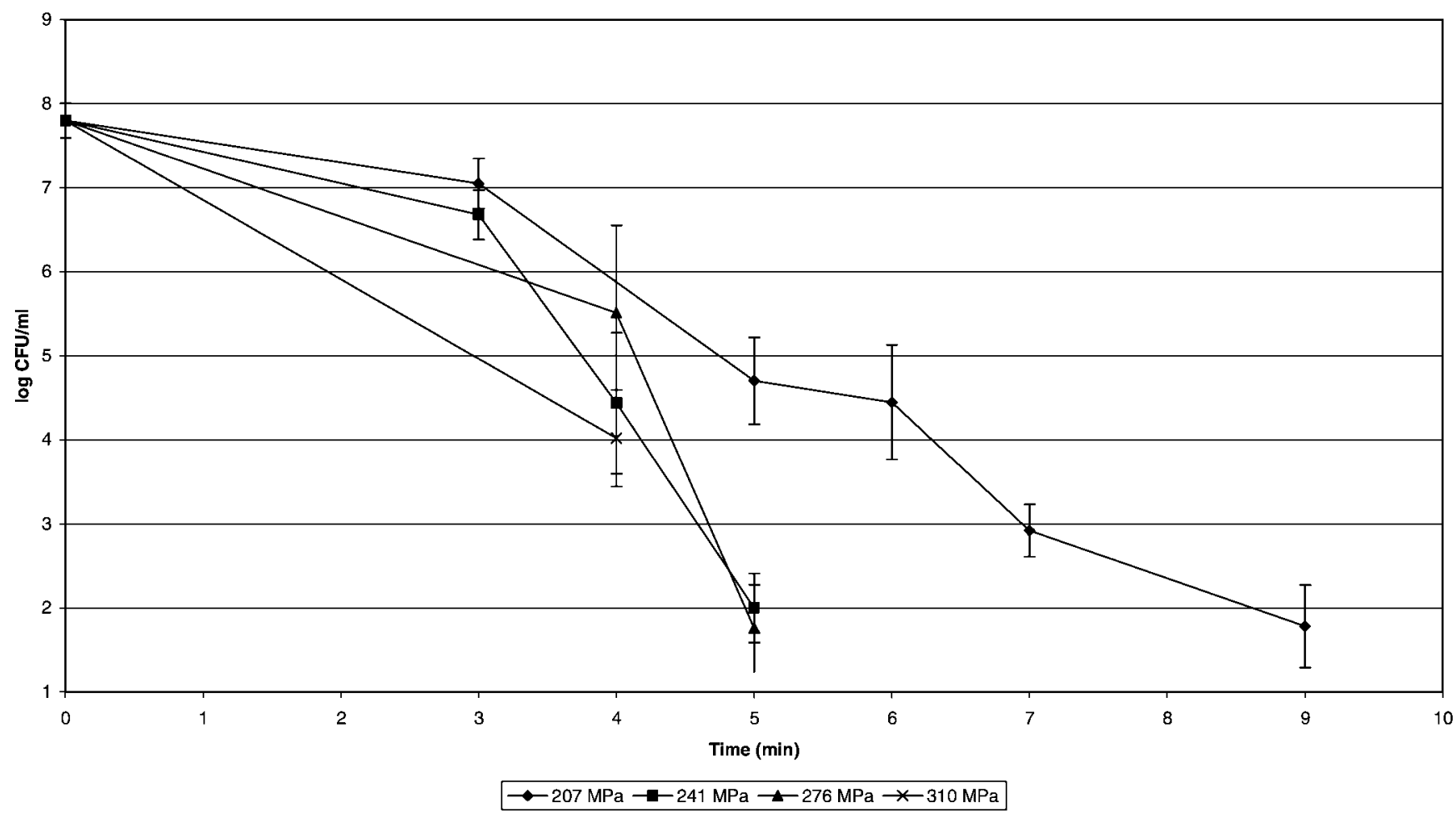

FIGURE 2. HPP inactivation of V. vulnificus MO-624 (clinical isolate) in PBS. Pressure come-up times are included in processing times.

min, which includes a 2-min pressure come-up time, $V$. vulnificus was reduced by more than $6.3 \log$ units, to nondetectable numbers (Fig. 2). Styles et al. (15) reported a 5$\log$ reduction for $V$. parahaemolyticus in PBS processed at $170 \mathrm{MPa}$ for $30 \mathrm{~min}$. V. parahaemolyticus and $V$. vulnificus in artificial seawater were reduced to nondetectable numbers at $250 \mathrm{MPa}$ for $15 \mathrm{~min}$ or $300 \mathrm{MPa}$ for $5 \mathrm{~min}(1)$. At $241 \mathrm{MPa}$ for $10 \mathrm{~min}$, two strains of $V$. parahaemolyticus in PBS were reduced by $7 \log$ units (2).

In the present study, a more than 6-log reduction was

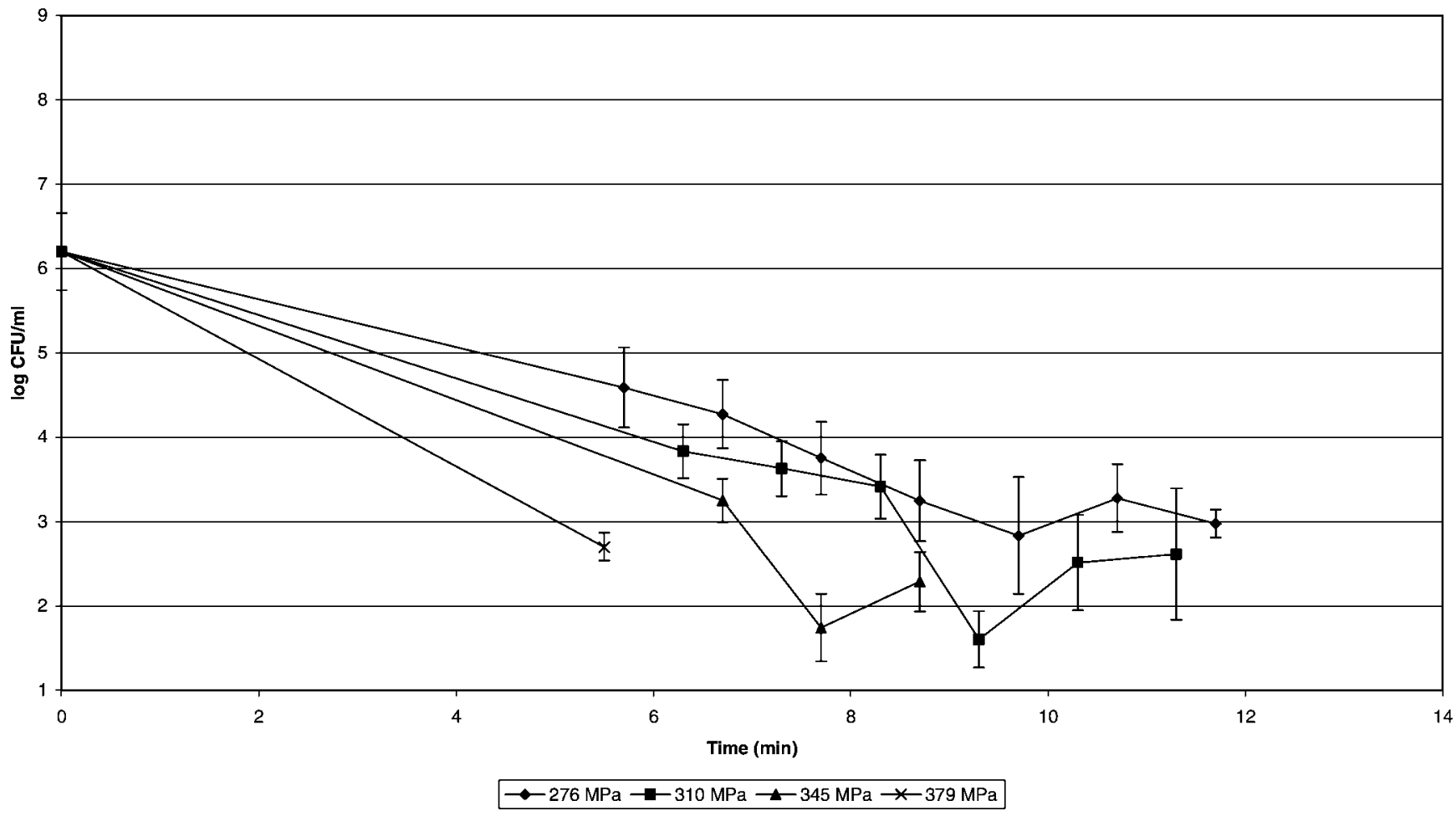

FIGURE 3. HPP inactivation of V. parahaemolyticus TX-2103 (serotype O3:K6) in oysters. Pressure come-up times are included in processing times. 
TABLE 2. D-values for V. parahaemolyticus TX-2103 (serotype O3:K6) and V. vulnificus MO-624 (clinical isolate) in PBS and in whole oysters

$D$-values $(\min )^{a}$

\begin{tabular}{cccc} 
& & & \\
\cline { 2 - 3 } $\begin{array}{c}\text { Pressure } \\
(\mathrm{MPa})\end{array}$ & PBS & -values (min) & \\
\cline { 2 - 3 } & V. parahaemolyticus & V. vulnificus & $\begin{array}{c}\text { Oyster } \\
(\text { V. parahaemolyticus })\end{array}$ \\
\hline 207 & $2.9 \pm 0.245$ & $1.4 \pm 0.171$ & NC \\
241 & $1.6 \pm 0.132$ & $0.8 \pm 0.136$ & NC \\
276 & $1.1 \pm 0.074$ & $0.8 \pm 0.145$ & $3.4 \pm 0.11$ \\
310 & $0.9 \pm 0.085$ & NC & $2.8 \pm 0.269$ \\
345 & NC & NC & $2.0 \pm 0.166$ \\
\hline
\end{tabular}

${ }^{a}$ Values are means \pm standard deviations (three to six replications conducted in duplicate). Due to rapid reduction in numbers of $V$. vulnificus in oysters at all pressures, $D$-values for $V$. vulnificus could not be calculated. NC, not calculated.

achieved at $241 \mathrm{MPa}$ for $11 \mathrm{~min}$, which included a 3-min pressure come-up time (Fig. 1). In comparison, Cook (3) reported that the minimum times required at $200 \mathrm{MPa}$ for a 5-log reduction in $V$. parahaemolyticus and $V$. vulnificus were approximately 7 and 2 min, respectively. Calik et al. (2) reported that $V$. parahaemolyticus was reduced by $8 \log$ units to nondetectable numbers after processing at $345 \mathrm{MPa}$ for $50 \mathrm{~s}$ or at 310 for $150 \mathrm{~s}$. When comparing multiple strains of $V$. vulnificus, $V$. parahaemolyticus, and $V$. cholerae non-O1, strains of $V$. vulnificus in PBS were the most pressure sensitive at $200 \mathrm{MPa}$ (3). Six Vibrio species including V. vulnificus responded similarly at 200 and 250 $\operatorname{MPa}(1)$.

Inoculated oysters. No differences in cell survival were attributable to shipping and handling. Initial V. parahaemolyticus and $V$. vulnificus populations in inoculated oysters were 6.2 and $6.7 \log \mathrm{CFU} / \mathrm{g}$, respectively. In oysters, V. vulnificus MO-624 (clinical isolate) was more sensitive than $V$. parahaemolyticus TX-2103 (serotype O3:K3) at all pressures and times (Table 2). At 241 and $276 \mathrm{MPa}$ for 4 and $5 \mathrm{~min}$, which includes pressure come-up times of 3 and $4 \mathrm{~min}$, respectively, $V$. vulnificus in oysters was reduced to nondetectable numbers, a $>5.4-\log$ reduction (data not shown). However, Cook (3) reported that $V$. vulnificus in oysters and oyster homogenates was reduced to nondetectable numbers with a $>4.8-\log$ reduction after processing at $241 \mathrm{MPa}$ for $2 \mathrm{~min}$. The strain we used might be more pressure sensitive than strains used by Cook (3). V. vulnificus in oysters at $310 \mathrm{MPa}$ were reduced to nondetectable numbers $(>5.4 \mathrm{log})$ during the 4.5 -min pressure come-up time (data not shown). A 3- to 4-log reduction of V. parahaemolyticus occurred in oysters processed at 276, 310, and $345 \mathrm{MPa}$ for 8.7 to $11.7 \mathrm{~min}$, which included pressure come-up times of 5.7 to $6.7 \mathrm{~min}$, but the pathogen was not reduced to nondetectable numbers (Fig. 3). At pressures of $379 \mathrm{MPa}$ for $6.5 \mathrm{~min}$ (Fig. 3), which includes a 5.5-min pressure come-up time, and $586 \mathrm{MPa}$ (data not shown) during an 8-min pressure come-up time, $V$. parahaemolyticus was reduced to nondetectable numbers. Calik et al. (2) reported that environmental and clinical strains of $V$. parahaemolyticus survived after processing at $310 \mathrm{MPa}$ for 180 $\mathrm{s}$ but were reduced to nondetectable numbers after processing at $345 \mathrm{MPa}$ for $120 \mathrm{~s}$.

$D$-values for $V$. parahaemolyticus TX-2103 (serotype O3:K6) in oysters at 276 and $310 \mathrm{MPa}$ were three times greater than those in PBS (Table 2). Longer processing times were required to reduce $V$. parahaemolyticus in oysters compared with PBS, indicating that oyster meat may protect $V$. parahaemolyticus from the lethal effects of pressure. $D$-values for $V$. parahaemolyticus in PBS at 207 and $241 \mathrm{MPa}$ were approximately two times greater than those for V. vulnificus in PBS at 207 and $241 \mathrm{MPa}$ (Table 2). Because of the dramatic reduction in $V$. vulnificus MO-624 (clinical isolate) at all pressures, $D$-values for $V$. vulnificus in oysters could not be calculated.

The effects of the same pressures and processing times were slightly less pronounced for APCs than for V. parahaemolyticus or $V$. vulnificus (Table 3). Recovery of $V$. par-

TABLE 3. Log reduction of APCs in inoculated oysters processed with HPP

\begin{tabular}{|c|c|c|c|c|}
\hline \multirow{2}{*}{$\begin{array}{l}\text { Pressure } \\
(\mathrm{MPa})\end{array}$} & \multicolumn{2}{|c|}{$\begin{array}{l}\text { Processing time }(\mathrm{min}) \text { for oysters } \\
\text { inoculated with: }\end{array}$} & \multicolumn{2}{|c|}{$\begin{array}{c}\text { Log reductions in APCs }(\mathrm{CFU} / \mathrm{g}) \text { for oysters } \\
\text { inoculated with: }\end{array}$} \\
\hline & V. parahaemolyticus & V. vulnificus & V. parahaemolyticus & V. vulnificus \\
\hline 241 & ND & 2 & ND & 4.3 \\
\hline 276 & 6 & 2 & 5.7 & 3.7 \\
\hline 310 & 5 & 2 & 4.3 & 3.6 \\
\hline 345 & 2 & 1 & 4.3 & 4.6 \\
\hline 379 & 1 & 0 & 4.8 & $>6.5$ \\
\hline 586 & 0 & 0 & $>5.5$ & $>6.5$ \\
\hline
\end{tabular}

${ }^{a}$ Three to six replications for each condition were conducted in duplicate. ND, not determined. 
ahaemolyticus and $V$. vulnificus was greater on nonselective TSAS agar than on selective TCBS or mCPC agars. Similar results were reported for high-pressure studies with Listeria monocytogenes in raw milk (8). APCs were not reduced to nondetectable numbers at most pressure settings except the highest pressure of $586 \mathrm{MPa}$ (Table 3). López-Caballero et al. (11) reported that $400 \mathrm{MPa}$ for $10 \mathrm{~min}$ at $7^{\circ} \mathrm{C}$ reduced total viable microorganisms in oysters to nondetactable numbers. The shelf life of pressure-treated oysters (APCs exceeding $10^{6} \mathrm{CFU} / \mathrm{g}$ ) at 207 to $311 \mathrm{MPa}$ was 20 days at $4^{\circ} \mathrm{C}$, whereas that of controls was 9 days $(9)$.

The results of this study indicate that high-pressure treatment is effective for reducing populations of $V$. parahaemolyticus TX-2103 (serotype O3:K6) and V. vulnificus MO-624 (clinical isolate). The longer pressure come-up times in this study did not significantly shorten processing times required to destroy $V$. parahaemolyticus or $V$. vulnificus compared with results obtained by Calik et al. (2) and Cook (3). Strains of V. parahaemolyticus O3:K6 are the most pressure resistant of the Vibrio species tested (e.g., $V$. cholerae, V. parahaemolyticus, and V. vulnificus) (3). In this study, a $>5-\log$ reduction of $V$. parahaemolyticus in oysters was achieved at $379 \mathrm{MPa}$ within $6.5 \mathrm{~min}$, which included a 5.5-min pressure come-up time (Fig. 3). A $>5.5$ $\log$ reduction of $V$. vulnificus in oysters was achieved at $241 \mathrm{MPa}$ within $5 \mathrm{~min}$, which included a 4-min pressure come-up time (data not shown).

\section{ACKNOWLEDGMENTS}

Funding for this project was provided by the National Sea Grant Gulf Oyster Industry Program (529458-VA-G1-99-1) and was administered through the Virginia Graduate Marine Science Consortium and Virginia Sea Grant College Program. Clinical isolates of V. parahaemolyticus TX-2103 (serotype O3:K6) and V. vulnificus MO-624 were obtained from the U.S. Food and Drug Administration (Dauphin Island, Ala.).

\section{REFERENCES}

1. Berlin, D. L., D. S. Herson, D. T. Hicks, and D. G. Hoover. 1999. Response of pathogenic Vibrio species to high hydrostatic pressure. Appl. Environ. Microbiol. 65:2776-2780.
2. Calik, H., M. T. Morrissey, P. W. Reno, and H. An. 2002. Effect of high-pressure processing on Vibrio parahaemolyticus strains in pure culture and Pacific oysters. J. Food Sci. 67:1506-1510.

3. Cook, D. W. 2003. Sensitivity of Vibrio species in phosphate-buffered saline and in oysters to high-pressure processing. J. Food Prot. 66:2276-2282.

4. Cook, D. W., P. O'Leary, J. C. Hunsucker, E. M. Sloan, J. C. Bowers, R. J. Blodgett, and A. DePaola. 2002. Vibrio vulnificus and Vibrio parahaemolyticus in U.S. retail shell oysters: a national survey from June 1998 to July 1999. J. Food Prot. 65:79-87.

5. Daniels, N. A., B. Ray, A. Easton, N. Marano, E. Kahn, A. L. McShan, L. Del Rosario, T. Baldwin, M. A. Kingsley, N. D. Puhr, J. G. Wells, and E. J. Angulo. 2000. Emergence of a new Vibrio parahaemolyticus serotype in raw oysters. JAMA 284:1541-1545.

6. DePaola, A., L. H. Hopkins, and R. M. McPhearson. 1988. Evaluation of four methods for enumeration of $V$. parahaemolyticus. Appl. Environ. Microbiol. 54:617-618.

7. DePaola, A., C. A. Kaysner, J. Bowers, and D. W. Cook. 2000. Environmental investigations of Vibrio parahaemolyticus in oysters following outbreaks in Washington, Texas and New York (19971998). Appl. Environ. Microbiol. 66:4649-4654.

8. Erkmen, O., and C. Dogan. 2004. Effects of ultra high hydrostatic pressure on Listeria monocytogenes and natural flora in broth, milk and fruit juices. Int. J. Food Sci. Technol. 39:91-97.

9. He, H., R. M. Adams, D. F. Farkas, and M. T. Morrissey. 2002. Use of high-pressure processing for oyster shucking and shelf-life extension. J. Food Sci. 67:640-645.

10. Hugas, M., M. Garriga, and J. M. Monfort. 2002. New mild technologies in meat processing: high pressure as a model technology. Meat Sci. 62:359-371.

11. López-Caballero, M. E., M. Pérez-Mateos, P. Montero, and A. J. Borderías. 2000. Oyster preservation by high-pressure treatment. $J$. Food Prot. 63:196-201.

12. Mead, P. S., L. Slutsker, V. Dietz, L. F. McGaig, J. S. Bresee, C. Shapiro, P. M. Griffin, and R. V. Tauxe. 1999. Food-related illness and death in the United States. Emerg. Infect. Dis. 5:607-625.

13. San Martin, M. F., G. V. Barbosa-Cánova, and B. G. Swanson. 2002. Food processing by high hydrostatic pressure. Crit. Rev. Food Sci. Nutr. 42:627-645.

14. Shapiro, R. L., S. Altekruse, L. Hutwagner, R. Bishop, R. Hammond, S. Wilson, B. Ray, S. Thompson, R. V. Tauxe, P. M. Griffin, and the Vibrio Working Group. 1998. The role of Gulf Coast oysters harvested in warmer months in Vibrio vulnificus infections in the United States, 1988-1996. J. Infect. Dis. 178:752-759.

15. Styles, M. F., D. G. Hoover, and D. F. Farkas. 1991. Response of Listeria monocytogenes and Vibrio parahaemolyticus to high hydrostatic pressure. J. Food Sci. 56:1404-1407. 